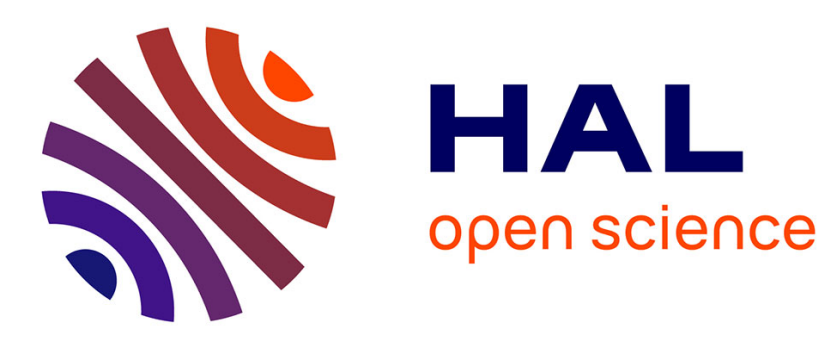

\title{
Concentration du rayonnement solaire: analyse et évaluation des défauts de réglage de facettes réfléchissantes
}

\author{
F. Henault, C. Royere
}

\section{- To cite this version:}

F. Henault, C. Royere. Concentration du rayonnement solaire: analyse et évaluation des défauts de réglage de facettes réfléchissantes. Revue de Physique Appliquée, 1989, 24 (5), pp.563-576. 10.1051/rphysap:01989002405056300 . jpa-00246082

\section{HAL Id: jpa-00246082 https://hal.science/jpa-00246082}

Submitted on 1 Jan 1989

HAL is a multi-disciplinary open access archive for the deposit and dissemination of scientific research documents, whether they are published or not. The documents may come from teaching and research institutions in France or abroad, or from public or private research centers.
L'archive ouverte pluridisciplinaire HAL, est destinée au dépôt et à la diffusion de documents scientifiques de niveau recherche, publiés ou non, émanant des établissements d'enseignement et de recherche français ou étrangers, des laboratoires publics ou privés. 


\title{
Concentration du rayonnement solaire : analyse et évaluation des défauts de réglage de facettes réfléchissantes
}

\author{
F. Henault et C. Royere \\ Institut de Science et Génie des Matériaux et Procédés, CNRS, BP N 5, Odeillo, 66120 Font-Romeu, France
}

(Reçu le 13 janvier 1988, révisé le 8 décembre, accepté le 6 février 1989)

\begin{abstract}
Résumé. - Les défauts de réglage des facettes réflectrices qui équipent les grandes installations solaires sont des paramètres primordiaux pour la modélisation des répartitions des flux réfléchis. Leur caractérisation peut être effectuée par l'examen direct des répartitions de luminance apparentes sur les surfaces réflectrices et observées d'un point situé au voisinage du point focal de l'installation. On décrit ici le cadre d'une approximation visant à exprimer l'équation des courbes isoluminance idéales. Puis les écarts typiques des surfaces réflectrices des installations solaires sont introduits et on établit les relations permettant de remonter aux défauts de réglage. Des méthodes applicables aux installations à simple réflexion du type centrale à tour, et à double réflexion de type four solaire sont décrites et on donne quelques résultats préalables aux campagnes de mesure.
\end{abstract}

\begin{abstract}
The alignment errors of the reflecting facets used in large scale solar point focusing facilities are prime order parameters for computer modeling maps of the reflected fluxes. They can be determined by direct observation of the apparent radiances distributions on the reflecting surfaces sighted from a point in the vicinity of the focal point of the facility. Herein we describe the approximation leading to establishing the equation of ideal isoradiance curves. Then the current typical deviations of the reflecting surfaces in the solar facilities are introduced and the equations allowing to get in turn the alignment errors are established. Methods adapted to simple reflection (solar tower concept type) and double reflection (solar furnace concept type) are presented and some preliminary experimental results are given prior to an extensive campaign of measurements.
\end{abstract}

\section{Introduction.}

L'évaluation prévisionelle des performances en densité de flux, ou éclairement, des grandes installations solaires à surfaces réfléchissantes est un problème complexe qui nécessite la connaissance de nombreux paramètres. Certains d'entre eux caractérisant les défauts de surface et de réglage des miroirs utilisés sur l'installation, sont encore très mal connus. Notre but est de préciser les paramètres d'erreur dont la connaissance est indispensable et de présenter quelques méthodes d'évaluation possibles. Celles-ci sont toutes basées sur les principes exposés par Brumleve et Gibson [1] : au lieu de caractériser, par exemple à l'aide d'une caméra, ou par calorimétrie ou radiométrie, les répartitions de densité de flux formées dans un plan récepteur, et d'en tirer des informations globales sur la qualité des surfaces réflectrices, la caméra sera placée au milieu du plan récepteur, et observera directement (du plan de réception) les images du soleil, ou répartitions de luminance, issues de la surface réfléchissante (Fig. 1). Ainsi des
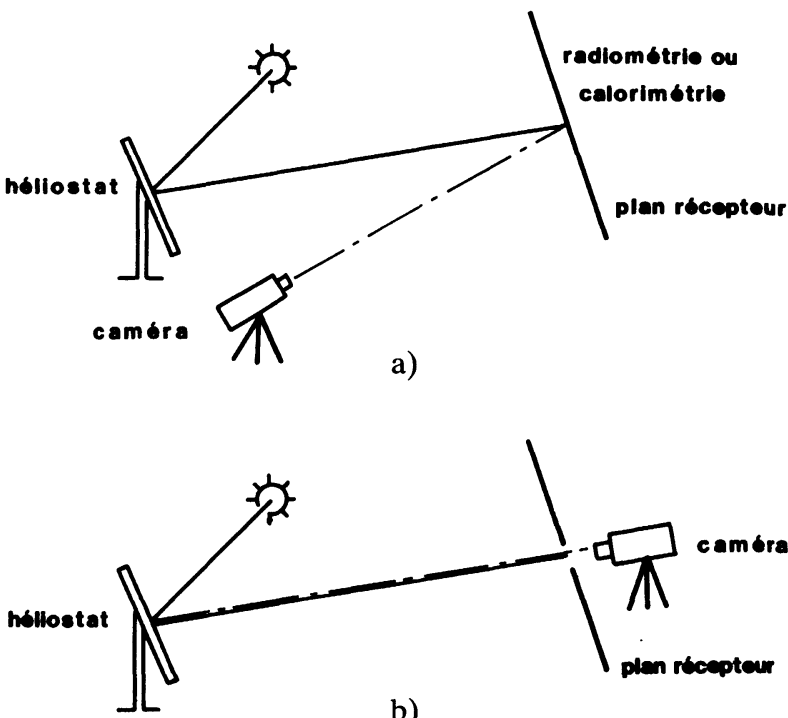

b)

Fig. 1. - Mesures de densité de flux (a) et mesures de luminance (b).

[Mapping irradiances (a) and radiances (b).] 
informations locales pourront être obtenues. Après avoir examiné d'un point de vue théorique, quelles devraient être les répartitions de luminance idéales, nous préciserons l'influence des défauts de réglage des miroirs sur ces répartitions, et proposerons, en conséquence, quelques méthodes d'estimation des déréglages et de contrôle de la qualité des surfaces réflectrices.

Trois types de surfaces à facettes seront considérées ici : les héliostats plans et les concentrateurs fixes d'une installation à double réflexion, du type four solaire, et les héliostats focalisants d'une installation à simple réflexion du type centrale à tour. En fait nous nous intéresserons plus particulièrement au four solaire de $1000 \mathrm{~kW}$ d'Odeillo et à la centrale solaire expérimentale Themis.

Présentation théorique, formulation intégrale de la densité de flux.

L'expression de la densité de flux $E\left(\mathrm{M}^{\prime}\right)$, formée en un point $\mathbf{M}^{\prime}$, par une facette réflectrice quelconque éclairée par le soleil est déduite de la loi de l'étendue géométrique [2,3]. Avant de l'appliquer, il est nécessaire de définir soigneusement les repères de travail.

CHOIX DES REPÈRES. - Le point $\mathbf{M}^{\prime}$ appartient au plan récepteur $\left(\mathrm{P}^{\prime}\right)$ d'origine $\mathrm{O}^{\prime}$, auquel est lié le repère $\mathrm{R}^{\prime}\left(\mathrm{O}^{\prime} X^{\prime} Y^{\prime} Z^{\prime}\right)$ (Fig. 2). L'axe $\mathrm{O}^{\prime} X^{\prime}$ est dirigé par $\mathbf{N}^{\prime}$, vecteur unitaire normal à $\left(\mathrm{P}^{\prime}\right)$. Les axes $O^{\prime} Y^{\prime}$ et $O^{\prime} Z^{\prime}$ appartiennent au plan récepteur $\left(\mathrm{P}^{\prime}\right)$, et on impose que $\mathrm{O}^{\prime} Y^{\prime}$ soit horizontal. Les coordonnées de $\mathrm{M}^{\prime}$ seront notées $\left(\mathrm{O}, Y^{\prime}, Z^{\prime}\right)$ dans ce repère.

La facette réflectrice considérée est rapportée au repère $\mathrm{R}_{0 i}\left(\mathrm{O}_{i} X_{0 i} Y_{0 i} Z_{0 i}\right)$ (Fig. 2). Le point $\mathrm{O}_{i}$ est le centre de la facette, l'axe $\mathrm{O}_{i} X_{0 i}$ est dirigé par $\mathbf{N}_{0 i}$, normale unitaire à la facette en $\mathrm{O}_{i}$ et les axes $\mathrm{O}_{i} Y_{0 i}$ et $\mathrm{O}_{i} Z_{0 i}$ sont parallèles aux contours du miroir (qui sont rectangulaires à Odeillo comme à Themis). Les coordonnées d'une point $\mathrm{P}$ de la facette réflectrice sont notées $\left(X_{\mathrm{p}}, Y_{\mathrm{p}}, Z_{\mathrm{p}}\right)$ dans $\mathrm{R}_{0 i}$.

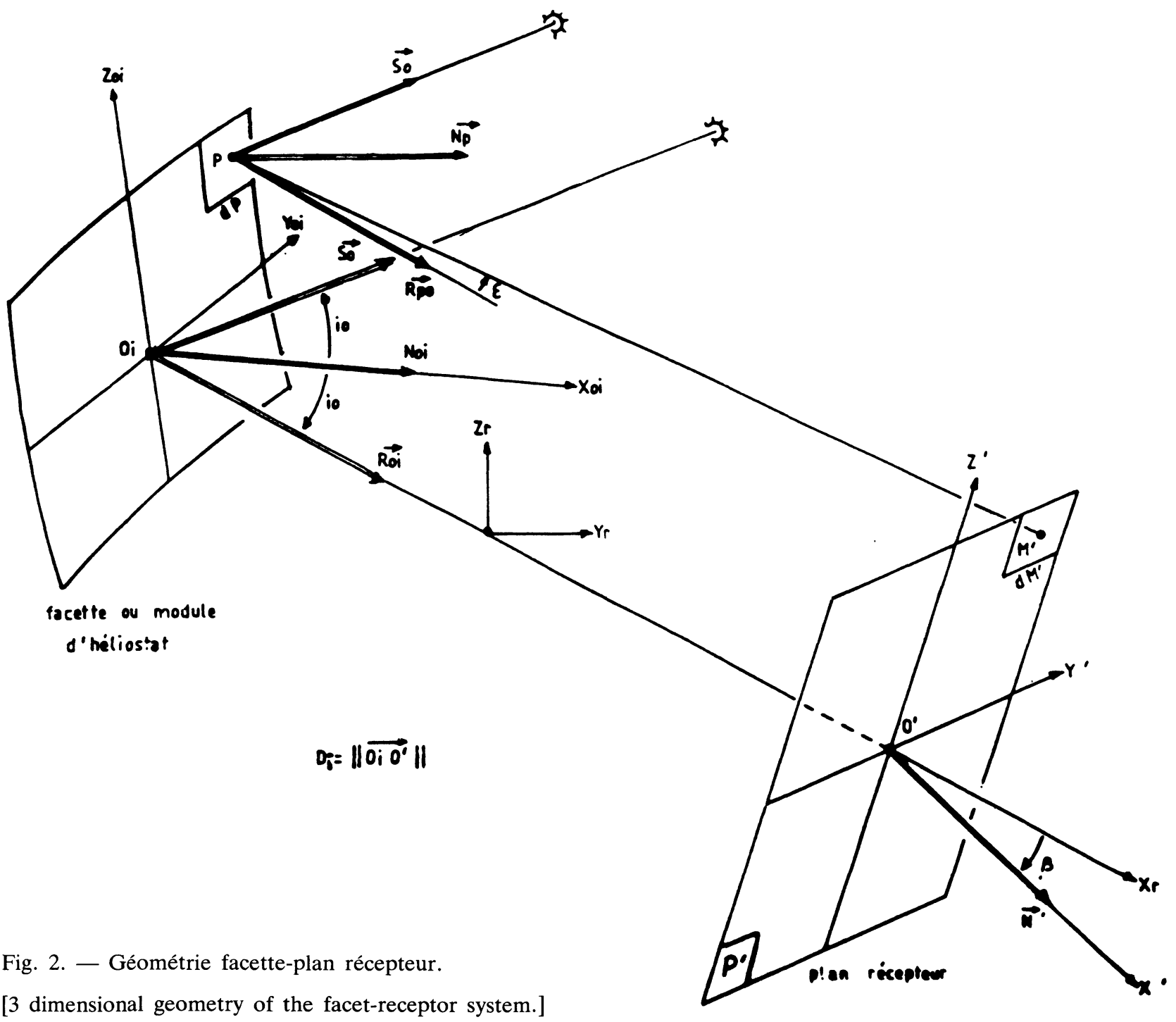

[3 dimensional geometry of the facet-receptor system.] 
Le repère principal, dans lequel sont effectués la plupart des calculs, est le repère $\mathrm{R}_{\mathrm{r}}\left(\mathrm{O}^{\prime} X_{\mathrm{r}} Y_{\mathrm{r}} Z_{\mathrm{r}}\right)$ (Fig. 2). L'axe $\mathrm{O}^{\prime} X_{\mathrm{r}}$ est confondu avec la droite $\mathrm{O}_{i} \mathrm{O}^{\prime}$, qui est le plus souvent le rayon principal réfléchi en $\mathrm{O}_{i}$, dirigé par $\mathbf{R}_{0 i} \cdot \mathbf{R}_{0 i}$ est déduit de $S_{0}$, vecteur unitaire pointé sur le centre du soleil, et de $\mathbf{N}_{0 i}$ par la loi de Descartes pour la réflexion des rayons lumineux (voir la relation (6)). Par ailleurs, l'axe $\mathrm{O}^{\prime} Y_{\mathrm{r}}$ est horizontal.

Lorsque la facette réflectrice est montée sur un héliostat, il est nécessaire d'adjoindre un quatrième repère $\mathrm{R}_{0}\left(\mathrm{O}, X_{0} Y_{0} Z_{0}\right)$ (Fig. 3), où $\mathrm{O}$ est le centre de l'héliostat, que l'on suppose confondu avec son centre de rotation mécanique, et où l'axe $\mathrm{O} X_{0}$ est dirigé par $\mathbf{N}_{0}$, normale à l'héliostat en $\mathrm{O}$. L'axe $O Y_{0}$ est toujours horizontal, les héliostats considérés étant équipés d'une monture altazimutale. Les coordonnées du centre $\mathrm{O}_{i}$ de la facette réflectrice considérée sont notées $\left(X_{0 i}, Y_{0 i}, Z_{0 i}\right)$ dans $\mathrm{R}_{0}$.

EXPRESSION EXACTE DE LA DENSITÉ DE FLUX. Considérons un élément réflecteur $\mathrm{dP}$ centré sur le point $\mathrm{P}$ du miroir et un élément récepteur $\mathrm{dM}^{\prime}$ centré sur le point $\mathrm{M}^{\prime}$ du plan $\left(\mathrm{P}^{\prime}\right)$. La quantité de flux $\delta^{2} \phi$ quittant dP pour atteindre $\mathrm{dM}^{\prime}$, est, conformément à la loi de l'étendue géométrique :

$$
\delta^{2} \phi=L_{\mathrm{r}}\left(\mathbf{P} \mathbf{M}^{\prime}\right)\left(\frac{\mathbf{P} \mathbf{M}^{\prime}}{\left\|\mathbf{P} \mathbf{M}^{\prime}\right\|} \cdot \mathbf{N}^{\prime}\right) \mathrm{dM}^{\prime} \mathrm{d} \Omega
$$

$L_{\mathrm{r}}\left(\mathbf{P} \mathbf{M}^{\prime}\right)$ est la luminance suivant le rayon $\mathbf{P M}^{\prime}$ et $\mathrm{d} \Omega$ est l'angle solide sous lequel est $\mathrm{vu}$, du point $\mathrm{M}^{\prime}$, l'élément dP (Fig. 2).

Si l'on note $\mathbf{N}_{\mathrm{p}}$ la normale unitaire à la surface $d u$ miroir en $\mathrm{P}$, et $\mathbf{R}_{\mathrm{p} 0}$ le vecteur qui dirige le rayon réfléchi en $\mathrm{P}$, et provenant du centre de soleil (Fig. 2), la contribution d'éclairement $\mathrm{d} E\left(\mathrm{M}^{\prime}\right)$ de l'élément réflecteur $\mathrm{dP}$ au point $\mathrm{M}^{\prime}$ s'écrit alors :

$$
\begin{aligned}
\mathrm{d} E\left(\mathbf{M}^{\prime}\right)=\frac{\delta^{2} \phi}{\mathrm{dM}^{\prime}}= & L_{\mathrm{r}}\left(\mathbf{P} \mathbf{M}^{\prime}\right) \times \\
& \times \frac{\left(\mathbf{P} \mathbf{M}^{\prime} \cdot \mathbf{N}^{\prime}\right)\left(\mathbf{P M}^{\prime} \cdot \mathbf{N}_{\mathrm{p}}\right)}{\left\|\mathbf{P} \mathbf{M}^{\prime}\right\|^{4}} \mathrm{dP} .
\end{aligned}
$$

Le disque solaire présentant une répartition de luminance $L(\varepsilon)$ symétrique par rapport à son centre, $L_{\mathrm{r}}\left(\mathbf{P M}^{\prime}\right)$ ne dépend que de $\varepsilon$, angle entre $\mathbf{P M}^{\prime}$ et $\mathbf{R}_{\mathrm{p} 0}$ (Fig. 2), et du facteur de réflexion $R$ du miroir que l'on suppose homogène sur toute sa surface.

Alors, en effectuant une somme continue sur les points $\mathrm{P}$ de la facette réflectrice, puis une somme discrète sur les $n_{\mathrm{T}}$ facettes que comprend l'installation, on obtient l'expression la plus générale de la densité de flux :

$$
\begin{aligned}
E\left(\mathbf{M}^{\prime}\right) & =\sum_{i=1}^{n_{T} \text { facettcs }} R \times \\
& \times \iint_{\substack{\text { ième } \\
\text { facette }}} L(\varepsilon) \frac{\left(\mathbf{P M}^{\prime} \cdot \mathbf{N}^{\prime}\right)\left(\mathbf{P M}^{\prime} \cdot \mathbf{N}_{\mathrm{p}}\right)}{\left\|\mathbf{P M}^{\prime}\right\|^{4}} \mathrm{dP}
\end{aligned}
$$

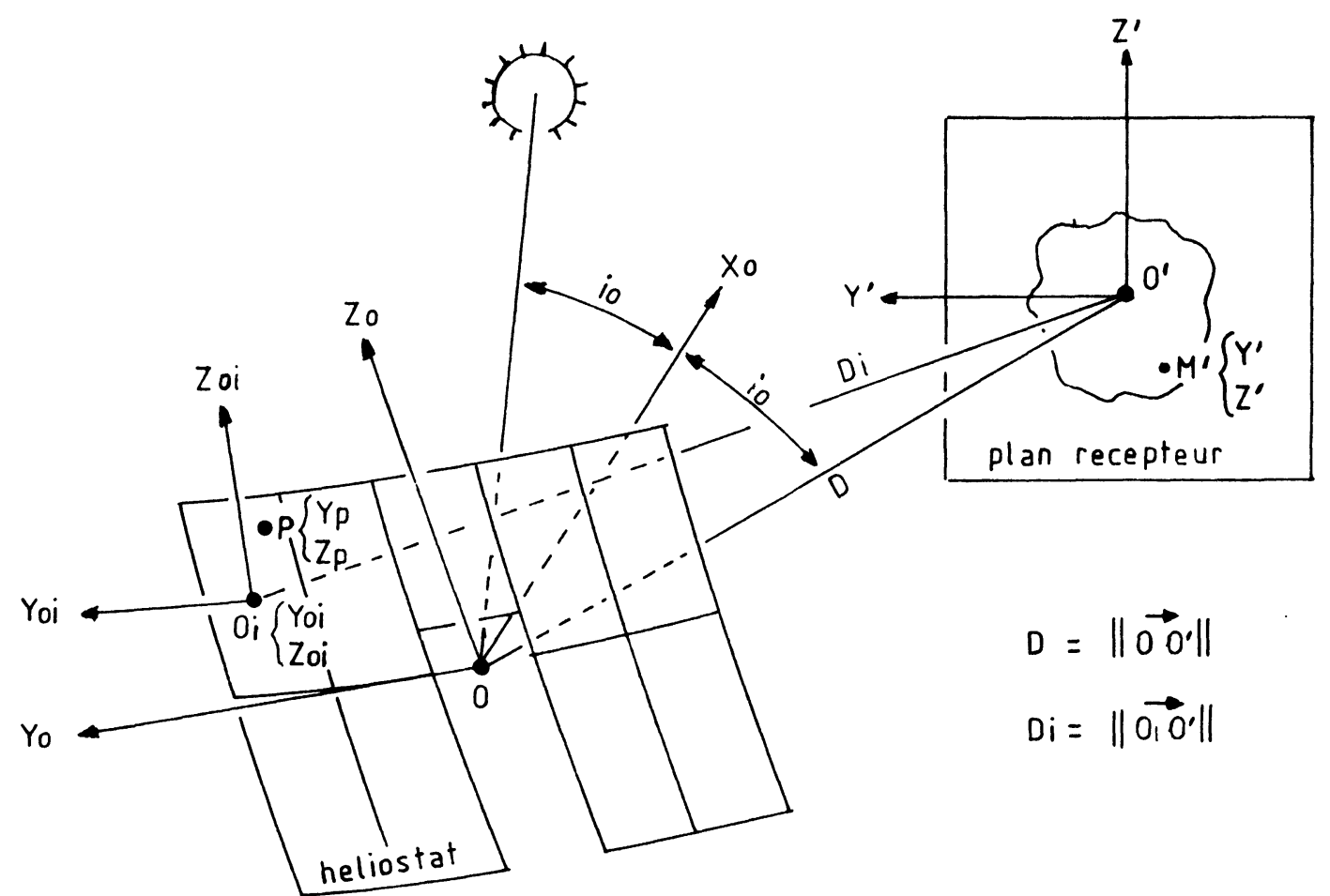

Fig. 3. - Les termes de l'approximation du premier ordre. Cas d'un héliostat.

[First order approximation terms. Case of a heliostat.] 
et la concentration atteinte au point $\mathrm{M}^{\prime}$ s'écrit :

$$
C\left(\mathrm{M}^{\prime}\right)=E\left(\mathrm{M}^{\prime}\right) / E_{0}
$$

où $E_{0}$ est l'éclairement solaire direct normal mesuré sur le site.

La relation (3) signifie que l'éclairement en $\mathrm{M}^{\prime}$ est la somme directionnelle des répartitions de luminance observées dans les miroirs. L'expression de $\varepsilon$ doit être explicitée. On l'obtient par :

$$
\varepsilon^{2} \approx \sin ^{2} \varepsilon=\frac{\left\|\mathbf{P M}^{\prime} \wedge \mathbf{R}_{\mathrm{p} 0}\right\|^{2}}{\left\|\mathbf{P M}^{\prime}\right\|^{2}}
$$

$\mathbf{R}_{\mathrm{p} 0}$ étant déterminé par la loi de Descartes pour la réflexion spéculaire :

$$
\mathbf{R}_{\mathrm{p} 0}=2\left(\mathbf{S}_{0} \cdot \mathbf{N}_{\mathrm{p}}\right) \mathbf{N}_{\mathrm{p}}-\mathbf{S}_{0} .
$$

Notre but est ici d'établir une expression approchée de l'angle $\varepsilon$ afin de déterminer les courbes isoluminance apparentes sur la surface du miroir à partir du point $\mathbf{M}^{\prime}$. Nous précisons maintenant les hypothèses de cette approximation, qui est du type premier ordre.

Expression de $\varepsilon$ dans le cadre d'une approximation du premier ordre.

Dans le cadre de cette approximation, nous utiliserons deux types d'infiniment petits : ceux qui rendent compte de la géométrie de l'ensemble surface réflectrice-plan récepteur, et ceux qui décrivent les défauts spécifiques des installations solaires multifacettes et multi-héliostats.

LES INFINIMENT PETITS « GÉOMÉTRIQUES ». - En règle générale, l'angle d'incidence moyen $i_{0}$ des rayons solaires sur la facette réflectrice, ou sur l'héliostat (Fig. 3) est non négligeable; il peut atteindre $35^{\circ}$ sur un concentrateur de four solaire, et plus de $50^{\circ}$ dans le cas d'héliostats focalisants pour centrale à tour. $i_{0}$ ne peut donc pas être choisi comme infiniment petit, et l'approximation décrite ici est différente de l'approximation de Gauss, même si elle repose sur le fait que l'ouverture angulaire des miroirs (ou des héliostats) considérés individuellement reste faible sur les deux installations étudiées $\left(1^{\circ}\right.$ au maximum pour le concentrateur du four de $1000 \mathrm{~kW}$ d'Odeillo, et $2,5^{\circ}$ pour les héliostats focalisants de la centrale Themis. L'hypothèse de base s'exprime ainsi : les dimensions des facettes réflectrices, des héliostats, et des répartitions de densité de flux qu'ils forment dans le plan récepteur $\left(\mathrm{P}^{\prime}\right)$, restent faibles devant les distances qui séparent les surfaces réflectrices du plan $\left(\mathrm{P}^{\prime}\right)$. Les infiniment petits du premier ordre choisis seront donc $Y^{\prime} / D_{i}$ et $Z^{\prime} / D_{i}, Y_{\mathrm{p}} / D_{i}$ et $Z_{\mathrm{p}} / D_{i}, Y_{0 i} / D_{i}$ et $Z_{0 i} / D_{i} . D_{i}$ sera le plus souvent la distance $\mathrm{O}_{i} \mathrm{O}^{\prime}$ mais pourra éventuellement être remplacé par la distance ${ }^{\prime} O^{\prime}$ qui reste du même ordre, dans le cas d'un héliostat.

Parallèlement, on suppose que le relief du miroir est connu et qu'il est décrit par une fonction caractéristique $f\left(Y_{\mathrm{p}}, Z_{\mathrm{p}}\right)$ telle que :

$$
X_{\mathrm{p}}=f\left(Y_{\mathrm{p}}, Z_{\mathrm{p}}\right) \text {. }
$$

On note les dérivées partielles de $f$ par rapport à $Y_{\mathrm{p}}$ et $Z_{\mathrm{p}}$ :

$$
\begin{aligned}
& f_{y}^{\prime}=\frac{\partial f}{\partial Y_{p}}\left(Y_{\mathrm{p}}, Z_{\mathrm{p}}\right) \\
& f_{z}^{\prime}=\frac{\partial f}{\partial Z_{\mathrm{p}}}\left(Y_{\mathrm{p}}, Z_{\mathrm{p}}\right) .
\end{aligned}
$$

$f_{y}^{\prime}$ et $f_{z}^{\prime}$ sont considérées elles aussi comme des infiniment petits du premier ordre en fonction de $Y_{\mathrm{p}} / D_{i}$ et $Z_{\mathrm{p}} / D_{i}$. On se limite donc, dans le cadre de cette approximation, à ne considérer que les courbures principales des facettes réflectrices; cela est néanmoins suffisant pour modéliser la plupart des reliefs de miroirs (ou d'héliostats) effectivement utilisés sur les grandes installations solaires, et il s'ensuit que les termes $X_{\mathrm{p}} / D_{i}$, (ainsi que les termes $\left.X_{0 i} / D_{i}\right)$, du deuxième ordre en $Y_{\mathrm{p}} / D_{i}$ et $Z_{\mathrm{p}} / D_{i}$, sont négligeables.

Dans l'expression de $\varepsilon$ interviendront également les coefficients de deux matrices caractéristiques de l'emplacement de la facette réflectrice sur l'installation ; on note :

$$
P=\left[\begin{array}{lll}
C_{11} & C_{12} & C_{13} \\
C_{21} & C_{22} & C_{23} \\
C_{31} & C_{32} & C_{33}
\end{array}\right]
$$

la matrice de passage du repère $R_{r}$ lié aux rayons réfléchis au repère $\mathrm{R}_{0 i}$ lié à la facette réflectrice, et :

$$
P^{\prime}=\left[\begin{array}{lll}
C^{\prime}{ }_{11} & C^{\prime}{ }_{12} & C^{\prime}{ }_{13} \\
C^{\prime}{ }_{21} & C^{\prime}{ }_{22} & C^{\prime}{ }_{23} \\
C^{\prime}{ }_{31} & C^{\prime}{ }_{32} & C^{\prime}{ }_{33}
\end{array}\right]
$$

la matrice de passage du repère $R_{r}$ au repère $R^{\prime}$ lié au plan récepteur. Les deux matrices $P$ et $P^{\prime}$ sont orthogonales droites.

Enfin, on introduit les variables intermédiaires suivantes :

$$
\begin{aligned}
& V_{\mathrm{p}}=C_{22} Y_{\mathrm{p}}+C_{23} Z_{\mathrm{p}} \\
& W_{\mathrm{p}}=C_{32} Y_{\mathrm{p}}+C_{33} Z_{\mathrm{p}} \\
& V^{\prime}=C_{22}^{\prime} Y^{\prime}+C_{23}^{\prime} Z^{\prime} \\
& W^{\prime}=C_{32}^{\prime} Y^{\prime}+C_{33}^{\prime} Z^{\prime}
\end{aligned}
$$

où $V_{\mathrm{p}}$ et $W_{\mathrm{p}}$ sont les coordonnées apparentes du point $\mathrm{P}$ du miroir dans le plan $\left(\mathrm{O}_{i} Y_{\mathrm{r}} Z_{\mathrm{r}}\right)$ normal aux rayons réfléchis, et $V^{\prime}$ et $W^{\prime}$ sont les coordonnées de $\mathrm{H}^{\prime}$, projection du point $\mathrm{M}^{\prime}$ dans le plan $\left(\mathrm{O}^{\prime} Y_{\mathrm{r}} Z_{\mathrm{r}}\right)$ (Fig. 4). Bien entendu $V^{\prime} / D_{i}, W^{\prime} / D_{i}, V_{\mathrm{p}} / D_{i}$ et 


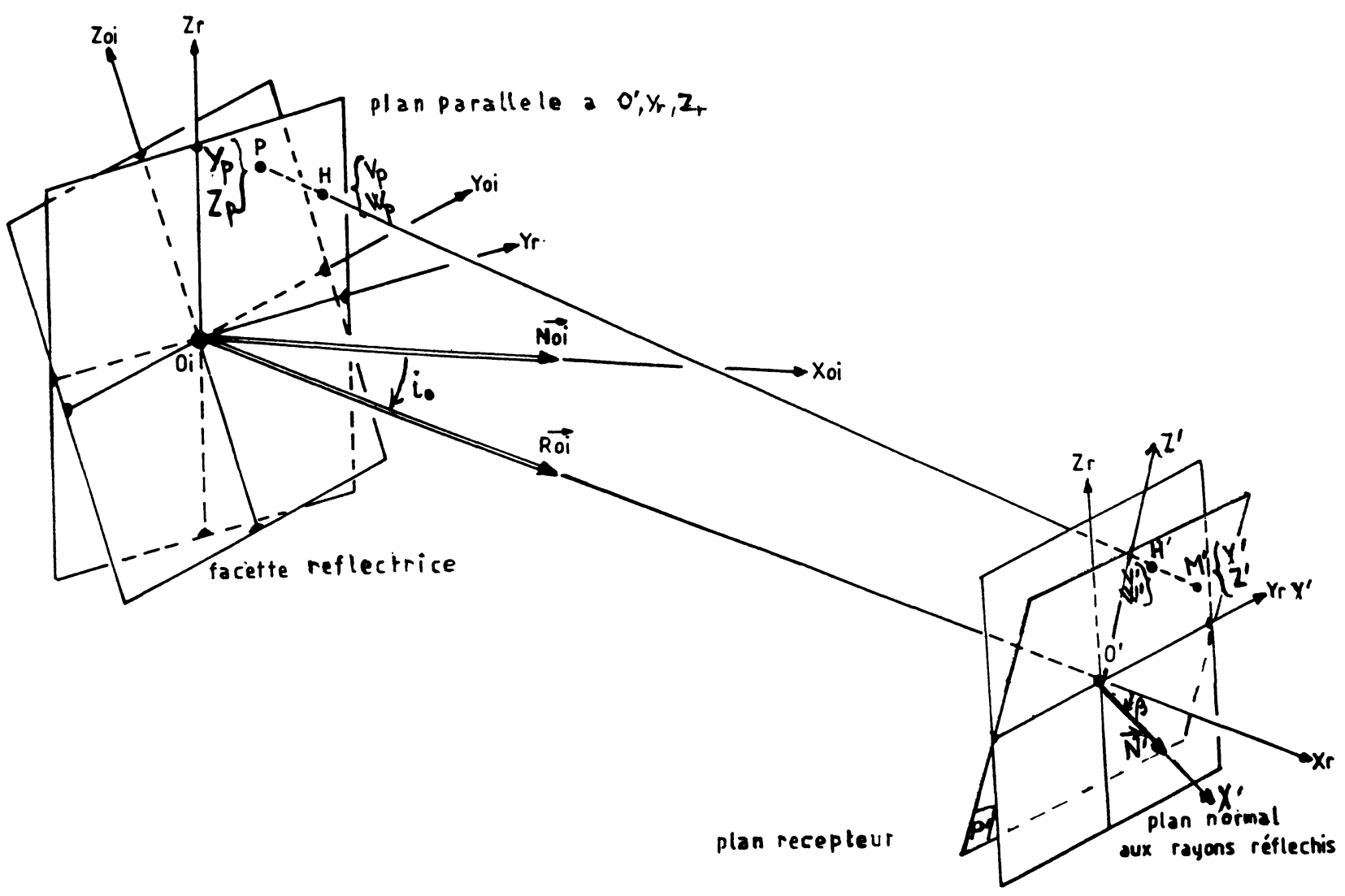

Fig. 4. - Relations entre coordonnées réelles et coordonnées projetées sur le plan normal aux rayons réfléchis.

[Relations between actual and projected coordinates (projection over the plane normal to the reflected beam).]

$W_{\mathrm{p}} / D_{i}$ sont des infiniment petits du premier ordre au même titre que ceux définis plus haut.

LES DÉFAUTS TYPIQUES DES INSTALLATIONS SOLAIRES. - De nombreuses études théoriques ou expérimentales [4-8] ont déjà été effectuées sur les sources d'erreur classiques des grandes installations solaires. On en distingue 4 types principaux (voir Fig. 5) :

1) les défauts microscopiques (parfois appelés " waviness »). Il s'agit de micro-imperfections typiques du verre, telles que bulles, pierres, stries, griffes, auxquelles s'ajoutent les défauts du film réflecteur, qui dépendent eux-mêmes de la technique de dépôt. L'effet global de ces erreurs est d'introduire une dispersion du faisceau réfléchi. Une méthode de réflectométrie [5] a été développée pour la détermination des indicatrices de réflexion engendrées par ces défauts ;

2) les défauts locaux de surface. Ils peuvent se présenter sous deux formes : défauts très locaux tels que dépressions, cratères, relief en «tôle ondulée ", ou défauts de courbure qui peuvent intéresser toute la surface de la facette. Leur origine est due à des facteurs aussi variés que les défauts de fabrication, les déformations des miroirs ou des structures qui les supportent sous l'effet de la gravité, du vent, du

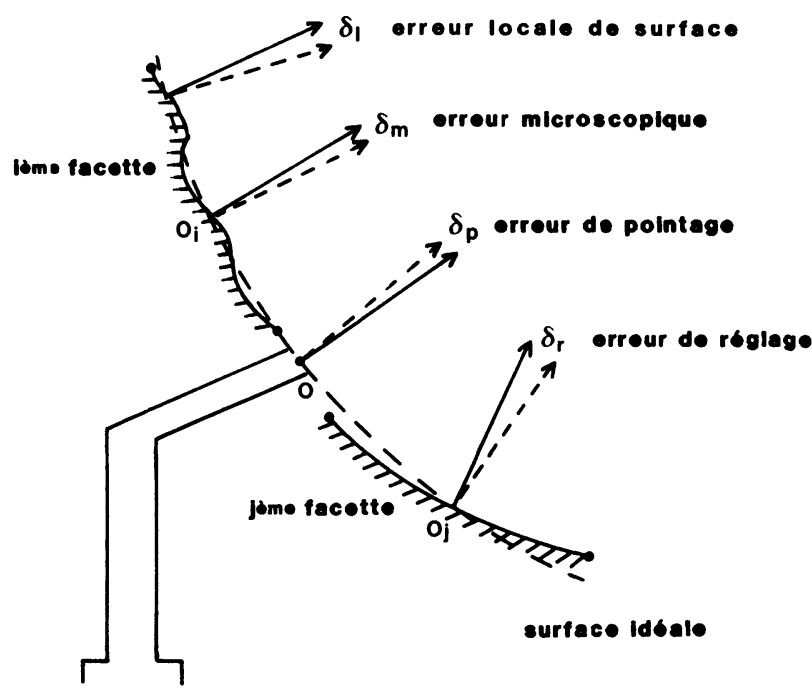

Fig. 5. - Erreurs typiques des surfaces réfléchissantes.

[Typical deviations of reflecting surfaces.]

poids de la neige, l'influence de gradients thermiques, etc... La meilleure méthode d'évaluation de ces défauts, vu leur ordre de grandeur relativement important, semble être la méthode par contact [6] ;

3) les défauts de pointage des héliostats [7-8]. Relativement bien connus, ils sont liés au système 
d'asservissement choisi pour l'héliostat. Des études ont montré que les distributions d'erreur mesurées dans le plan récepteur suivent en général des lois normales ;

4) les défauts de réglage des facettes. Ils apparaissent lors de la mise en place définitive des facettes sur leur infrastructure et sont étroitement liés à la méthode de réglage utilisée. Dans la plupart des cas d'installations ou de parties d'installation, il n'existe pas à notre connaissance de résultats expérimentaux sur ces défauts de réglage, ni même des méthodes de mesure qui permettraient de les déterminer.

Nous ne considérerons ici que les deux derniers types de défauts, car les défauts microscopiques et les défauts locaux de surface des miroirs peuvent être introduits dans l'expression de $\varepsilon$ de la même manière que leurs défauts de réglage. De plus, à l'échelle d'une facette réflectrice, ils n'en modifient les répartitions de luminance observées d'un point $\mathrm{M}^{\prime}$ que localement, alors que les dépointages et les déréglages les affectent globalement. On choisit donc comme infiniment petits du premier ordre $a_{\mathrm{p}}$ et $h_{\mathrm{p}}$, erreurs de pointage en azimut et en hauteur de l'héliostat, et $a_{\mathrm{r}}$ et $h_{\mathrm{r}}$ erreurs de réglage en azimut et en hauteur de la facette réflectrice. Ces erreurs sont dans la pratique de l'ordre de quelques milliradians.

EXPRESSION DE $\varepsilon$ EN FONCTION DU TYPE DE SURFACE RÉFLECTRICE ET DE SES DÉFAUTS. - Nous cherchons maintenant une expression de l'angle $\varepsilon$ approchée au premier ordre : pour cela, conformément à la relation (5), il est nécessaire de déterminer les composantes des vecteurs $\frac{\mathbf{P M}^{\prime}}{\left\|\mathbf{P M}^{\prime}\right\|}$ et $\mathbf{R}_{\mathrm{p} 0}$.

L'expression des composantes de $\mathbf{P M}^{\prime}$ dans le repère $R_{r}$ est obtenue grâce à la relation vectorielle :

$$
\mathbf{P} \mathbf{M}^{\prime}=\mathbf{O}^{\prime} \mathbf{M}^{\prime}-\mathbf{O}^{\prime} \mathbf{O}_{i}-\mathbf{O}_{i} \mathbf{P} .
$$

A partir de là, il est facile de montrer que :

$$
\frac{\mathbf{P M}^{\prime}}{\left\|\mathbf{P M}^{\prime}\right\|} \approx\left[\begin{array}{c}
1 \\
\left(V^{\prime}-V_{\mathrm{p}}\right) / D_{i} \\
\left(W^{\prime}-W_{\mathrm{p}}\right) / D_{i}
\end{array}\right]
$$

en éliminant tous les termes du second ordre dans les composantes du vecteur.

Pour calculer les composantes de $\mathbf{R}_{\mathrm{p} 0}$ on appliquera la relation (6) aux vecteurs $\mathbf{S}_{0}$ et $\mathbf{N}_{\mathrm{p}}$. Il est commode de raisonner d'abord dans le repère
$R_{0 i}$, et d'appliquer ensuite la matrice de passage $\mathrm{P}$ aux composantes ainsi obtenues.

On sait que le vecteur $S_{0}$ est symétrique du vecteur $\mathbf{R}_{0 i}$ (qui a pour composantes $(1,0,0)$ dans $\mathrm{R}_{\mathrm{r}}$ ) par rapport à $\mathbf{N}_{0 i}$, axe principal du repère $\mathrm{R}_{0 i}$. On en déduit que l'expression de $\mathrm{S}_{0}$ dans $\mathrm{R}_{0 i}$ est :

$$
\mathbf{S}_{0}=\left[\begin{array}{r}
C_{11} \\
-C_{12} \\
-C_{13}
\end{array}\right]
$$

Par ailleurs, l'expression de la normale $\mathbf{N}_{\mathrm{p}}$ à la surface réflectrice en $\mathrm{P}$ dans $\mathrm{R}_{0 i}$ s'obtient en additionnant tous les infiniment petits qui influent sur son orientation finale, c'est-à-dire, en fait, le relief du miroir et les défauts de pointage et de réglage :

$$
\mathbf{N}_{\mathrm{p}}=\left[\begin{array}{c}
1 \\
a_{\mathrm{p}}+a_{\mathrm{r}}-f_{y}^{\prime} \\
h_{\mathrm{p}}+h_{\mathrm{r}}-f_{z}^{\prime}
\end{array}\right] .
$$

En appliquant maintenant la relation (6) aux vecteurs, définis par (18) et (19), et en ne retenant que les termes du premier ordre, on trouve que :

$$
\mathbf{R}_{\mathrm{p} 0}=\left\{\begin{array}{r}
C_{11}-2 C_{12}\left(a_{\mathrm{p}}+a_{\mathrm{r}}-f_{y}^{\prime}\right) \\
-2 C_{13}\left(h_{\mathrm{p}}+h_{\mathrm{r}}-f_{z}^{\prime}\right) \\
C_{12}+2 C_{11}\left(a_{\mathrm{p}}+a_{\mathrm{r}}-f_{y}^{\prime}\right) \\
C_{13}+2 C_{11}\left(h_{\mathrm{p}}+h_{\mathrm{r}}-f_{z}^{\prime}\right)
\end{array}\right\}
$$

dans le repère $\mathrm{R}_{0 i}$.

Les coordonnées de $\mathbf{R}_{\mathrm{p} 0}$ dans $\mathbf{R}_{\mathrm{r}}$ s'expriment alors à l'aide de la matrice $P$, en utilisant les propriétés particulières de ce type de transformations : on sait en effet que la somme des carrés des éléments de chaque colonne (ou ligne) est égale à 1 , que la somme des produits des éléments correspondants de chaque colonne (ou ligne) est nulle, et que chaque élément $C_{i j}$ est égal à son cofacteur. Grâce à l'ensemble de ces relations, on arrive à simplifier considérablement l'expression de $\mathbf{R}_{\mathrm{p} 0}$ dans $\mathbf{R}_{\mathrm{r}}$ :

$\mathbf{R}_{\mathrm{p} 0}=\left\{\begin{array}{c}1 \\ 2 C_{33}\left(a_{\mathrm{p}}+a_{\mathrm{r}}-f_{y}^{\prime}\right)-2 C_{32}\left(h_{\mathrm{p}}+h_{\mathrm{r}}-f_{z}^{\prime}\right) \\ -2 C_{23}\left(a_{\mathrm{p}}+a_{\mathrm{r}}-f_{y}^{\prime}\right)+2 C_{22}\left(h_{\mathrm{p}}+h_{\mathrm{r}}-f_{z}^{\prime}\right)\end{array}\right\}$.

Des composantes de $\frac{\mathbf{P M}^{\prime}}{\left\|\mathbf{P M}^{\prime}\right\|}$ et $\mathbf{R}_{\mathrm{p} 0}$, on déduit enfin l'expression de $\varepsilon^{2}$ grâce à la relation (5). Au premier ordre :

$$
\begin{aligned}
\varepsilon^{2}=\left\{\frac{V_{\mathrm{p}}}{D_{i}}-2 C_{33} f_{y}^{\prime}+\right. & \left.2 C_{32} f_{z}^{\prime}+2 C_{33}\left(a_{\mathrm{p}}+a_{\mathrm{r}}\right)-2 C_{32}\left(h_{\mathrm{p}}+h_{\mathrm{r}}\right)-\frac{V^{\prime}}{D_{i}}\right\}^{2}+ \\
& +\left\{\frac{W_{\mathrm{p}}}{D_{i}}+2 C_{23} f_{y}^{\prime}-2 C_{22} f_{z}^{\prime}-2 C_{23}\left(a_{\mathrm{p}}+a_{\mathrm{r}}\right)+2 C_{22}\left(h_{\mathrm{p}}+h_{\mathrm{r}}\right)-\frac{W^{\prime}}{D_{i}}\right\}^{2} .
\end{aligned}
$$


Cette relation est également l'équation des courbes isoluminance apparentes du point $\mathbf{M}^{\prime}$ sur la surface réflectrice, avec $0 \leq \varepsilon \leq \varepsilon_{0}$, où $\varepsilon_{0}$ est le rayon angulaire du disque solaire ; c'est l'équation d'une conique puisque $f_{y}^{\prime}$ et $f_{z}^{\prime}$ sont proportionnelles à $Y_{\mathrm{p}}$ et $Z_{\mathrm{p}}$, et donc à $V_{\mathrm{p}}$ et $W_{\mathrm{p}}$ par l'intermédiaire des relations $(12,13)$ [9].

Dans le cas d'un héliostat plan ou focalisant, le calcul est mené de la même façon, mais, du fait de la différence d'emplacement entre $\mathrm{O}_{i}$ et $\mathrm{O}$ il apparaît, dans chacun des termes de l'équation, des expressions proportionnelles aux infiniment petits $Y_{0 i} / D_{i}$ et $Z_{0 i} / D_{i}$, venant s'ajouter aux termes en $a_{\mathrm{p}}$, $a_{\mathrm{r}}, h_{\mathrm{p}}$ et $h_{\mathrm{r}}$ [9]. Ces expressions, relativement complexes, ne seront pas données ici. Toutefois, leur seule conséquence sur les courbes isoluminance est de leur faire subir une translation dans le plan $\left(\mathrm{O}_{i} Y_{\mathrm{r}} Z_{\mathrm{r}}\right)$, translation qui sera caractérisée par le vecteur $\mathbf{T}_{0 i}$. A ce sujet, l'équation (22) nous permet de tirer trois enseignements, particulièrement importants dans la perspective de l'étude expérimentale des défauts de réglage.

1) Sauf cas de dégénérescence, les courbes isoluminance sont des ellipses, quel que soit le point d'observation $\mathrm{M}^{\prime}$, le relief du miroir, et le type de structure sur laquelle il est monté.

2) Lorsqu'on déplace le point d'observation $\mathbf{M}^{\prime}$, par l'intermédiaire des coordonnées $V^{\prime}$ et $W^{\prime}$, les courbes isoluminance apparentes sont décalées dans le plan de visée $\left(\mathrm{O}_{i} Y_{\mathrm{r}} Z_{\mathrm{r}}\right)$ mais leurs caractéristiques géométriques ne sont pas modifiées : les petits axes, les grands axes, et les orientations des ellipses images du disque solaire dans le plan $\left(\mathrm{O}_{i} Y_{\mathrm{r}} Z_{\mathrm{r}}\right)$ restent les mêmes.

Toute translation de ce type subie par les courbes isoluminance peut être décrite par un vecteur $\mathbf{T}_{\mathrm{M}^{\prime}}$.

3) Les défauts de réglage de la facette réflectrice $a_{\mathrm{r}}$ et $h_{\mathrm{r}}$ agissent de la même façon : les courbes isoluminance sont décalées mais non pas déformées. Un observateur placé au point $\mathbf{M}^{\prime}$ peut donc, en mesurant la translation $\mathbf{T}_{\mathrm{r}}$ subie par les ellipses images, à la surface d'un miroir, par rapport à leur position théorique (spécifiée par les vecteurs $\mathbf{T}_{\mathbf{M}^{\prime}}$ et $\mathbf{T}_{0 i}$ ), remonter aux erreurs de réglage $a_{\mathrm{r}}$ et $h_{\mathrm{r}}$ par inversion de relations linéaires déduites de l'équation (22) [9].

Enfin, les défauts de pointage des héliostats auront les mêmes conséquences, et leurs translations caractéristiques seront notées $\mathbf{T}_{\mathrm{p}}$. Toutefois, elles restent identiques pour tous les miroirs d'un même héliostat, et pourront donc facilement être éliminées lors du traitement statistique des défauts de réglage.

En résumé, la translation apparente $\mathbf{T}$ subie par les courbes isoluminance observables d'un point $\mathbf{M}^{\prime}$ sur une facette réflectrice quelconque peut être mise sous la forme d'une somme de translations :

$$
\mathbf{T}=\mathbf{T}_{\mathrm{M}^{\prime}}+\mathbf{T}_{\mathrm{r}}+\mathbf{T}_{\mathrm{p}}+\mathbf{T}_{0 i} .
$$

Or les translations suivant $\mathbf{T}_{\mathrm{M}^{\prime}}$ et $\mathbf{T}_{0 i}$ sont caractéristiques d'une géométrie surface réflectrice/point d'observation connue à l'avance, et les translations suivant $\mathbf{T}_{\mathrm{p}}$ sont les mêmes pour tous les miroirs d'un héliostat donné. Il est donc toujours possible de se servir des translations globales des courbes isoluminance pour remonter aux défauts de réglage $a_{\mathrm{r}}$ et $h_{\mathrm{r}}$ de la surface observée à partir du point $\mathbf{M}^{\prime}$, que ce soit d'une manière théorique [9], en extrayant des relations linéaires entre les composantes de $\mathbf{T}$ et $a_{\mathrm{r}}$ et $h_{\mathrm{r}}$ de l'équation (22), ou numérique [9] par simulation des répartitions de luminance sur ordinateur. Ces deux méthodes ont été employées avec un égal succès (voir paragraphe suivant).

La suite logique de cette partie théorique consiste maintenant à décrire quelques procédés expérimentaux simples qui permettent l'étude des défauts de réglage d'héliostats plans et focalisants in situ. Nous examinerons ensuite comment ces méthodes ont été adaptées au contrôle de la qualité de facettes réflectrices présentant des défauts de surface très marqués.

\section{Détermination expérimentale des sources d'erreur.}

Nous désirons enregistrer les répartitions de luminance observées, sur la surface réfléchissante, d'un point $\mathrm{M}^{\prime}$ situé dans le volume focal d'une installation solaire en fonctionnement. C'est une vue idéale qui ne pourra être réalisée effectivement. La zone d'observation sera en réalité un cercle de rayon a centré sur le point $\mathrm{M}^{\prime}$ (Fig. 6). De ce fait les rayons quittant le point $P$ pour atteindre cette zone ne présenteront pas tous la même luminance, puisqu'ils n'auront pas la même direction: il s'ensuit un brouillage des répartitions observées, qui est caractérisé par l'angle limite $r_{\theta}$, en dessous duquel les variations de luminance ne sont plus détectables. De même la résolution spatiale $r_{\mathrm{s}}$ qui caractérise la plus petite zone observable sur la facette doit être suffisamment fine pour effectuer des observations significatives. $r_{s}$ et $r_{\theta}$ dépendent bien sûr des moyens de visualisation employés. Nous avons utilisé un appareil photographique de $600 \mathrm{~mm}$ de focale, auquel nous avons adjoint suivant les cas un diaphragme supplémentaire devant sa pupille d'entrée. Si $D_{i}$ est la distance qui sépare la pupille d'entrée effective de l'objectif, de la surface étudiée, et $D$ est la distance de mise au point de l'appareil photographique, $r_{\mathrm{s}}$ et $r_{\theta}$ peuvent être respectivement considérées (Fig. 6) comme les réponses spatiales et directionnelles du point $\mathrm{M}^{\prime \prime}$ situé dans le plan image. On en déduit alors (Fig. 6) :

$$
\begin{aligned}
& r_{\mathrm{s}}=2 a \frac{D-D_{i}}{D} \\
& r_{\theta}=\frac{2 a}{D}
\end{aligned}
$$




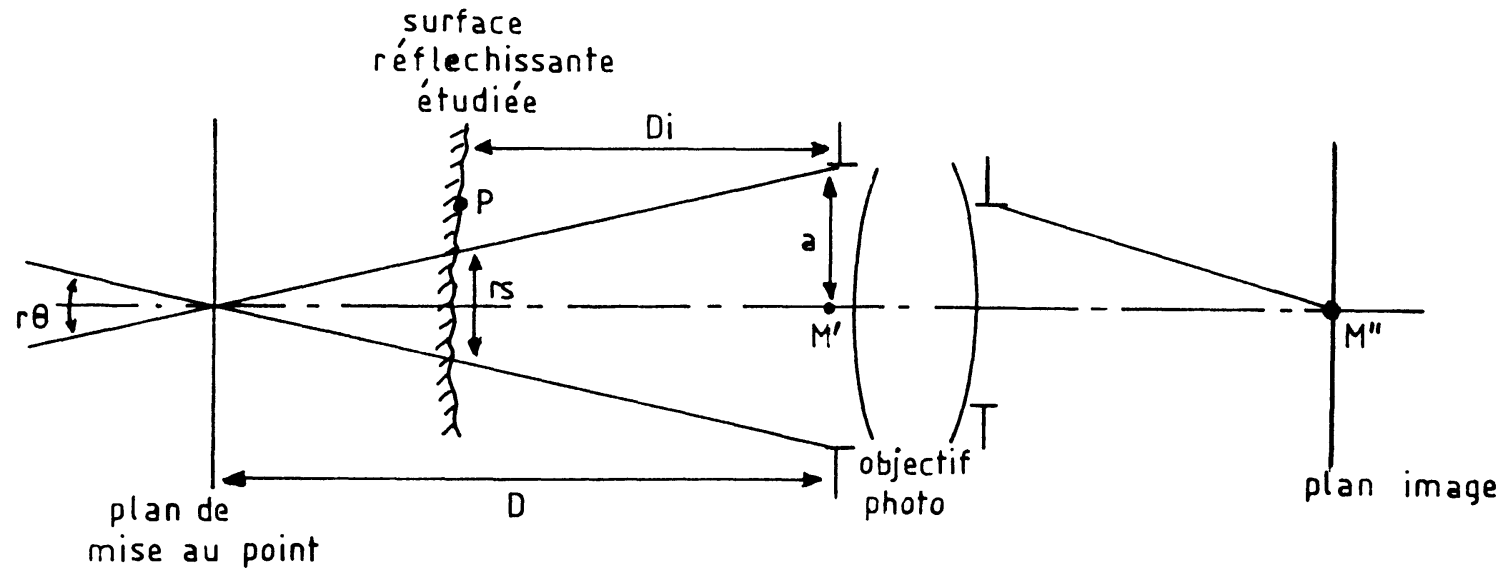

Fig. 6. - Résolution angulaire et spatiale $r_{\theta}$ et $r_{\mathrm{s}}$.

[Angular and spatial resolution $r_{\theta}$ and $r_{\mathrm{s}}$.]

$2 a$ est le diamètre de la pupille d'entrée effective de l'objectif. Il est intéressant de se ramener au plan image, afin de comparer avec les valeurs de résolution courantes des pellicules commerciales :

$$
\begin{aligned}
& r_{\mathrm{s}}^{\prime \prime}=2 f a \frac{D-D_{i}}{D D_{i}} \\
& r_{\theta}^{\prime \prime}=\frac{2 f a}{D} .
\end{aligned}
$$

Il faut également tenir compte du phénomène de diffraction lorsque $a$ devient très petit devant $D_{i}$. La résolution $r_{\mathrm{d}}^{\prime \prime}$ a pour expression approximative dans l'espace image :

$$
r_{\mathrm{d}}^{\prime \prime}=\frac{1,22 \lambda f}{a}
$$

où $\lambda$ est la longueur d'onde du rayonnement, et $f$ est la focale de l'objectif. On donne dans le tableau I $r_{\mathrm{s}}, r_{\theta}, r_{\mathrm{s}}^{\prime \prime}, r_{\theta}^{\prime \prime}$ et $r_{\mathrm{d}}^{\prime \prime}$ pour quelques valeurs choisies de $a$ et $D_{i}$, avec $\lambda=0,5 \mu \mathrm{m}, f=600 \mathrm{~mm}$, et en supposant vérifiée la relation :

$$
D=2 D_{i}
$$

relation qui établit le meilleur compromis entre résolution spatiale et résolution angulaire dans l'espace-image. Il s'ensuit que dans le cas d'héliostats, plans ou focalisants, situés à longue distance $\left(D_{i}>100 \mathrm{~m}\right)$, le plus petit diaphragme de l'objectif photographique permet d'obtenir des valeurs de résolutions satisfaisantes, tandis que dans le cas d'une facette de concentrateur située à $18 \mathrm{~m}$ de l'objectif, il est nécessaire de placer ce dernier derrière un petit trou, ce qui introduit évidemment beaucoup de diffraction, mais permet malgré tout d'obtenir des informations suffisantes sur la surface réflectrice.

ApPliCATION AUX HÉliostats PlANS. - Ces mesures ont été effectuées sur les héliostats plans du four
Tableau I. - Quelques valeurs des résolutions spatiale et angulaire pour des trous de diamètre $2 a=$ $7 \mathrm{~mm}$ et $16 \mathrm{~mm}$, et des distances $D_{i}=18 \mathrm{~m}$ et $100 \mathrm{~m}$. A titre de comparaison la résolution moyenne d'une pellicule est de $0,02 \mathrm{~mm}$.

[Some spatial and angular resolutions values for hole diameters $2 a=7 \mathrm{~mm}$ and $16 \mathrm{~mm}$ and distances $D_{i}=18 \mathrm{~m}$ and $100 \mathrm{~m}$. For comparison the mean resolution of a film is $0.02 \mathrm{~mm}$.]

\begin{tabular}{|l|l|l|l|l|}
\hline$a$ en mm & 3,5 & 3,5 & 8 & 8 \\
\hline$D_{i}$ en $\mathrm{m}$ & 18 & 100 & 18 & 100 \\
\hline$r_{\mathrm{s}}$ en $\mathrm{mm}$ & 3,5 & 3,5 & 8 & 8 \\
\hline$r_{\theta}$ en $\mathrm{mm}$ & 0,194 & 0,035 & 0,444 & 0,080 \\
\hline$r_{\mathrm{s}}^{\prime \prime}$ en $\mathrm{mm}$ & 0,117 & 0,021 & 0,267 & 0,048 \\
\hline$r_{\theta}^{\prime \prime}$ en mrad & 0,117 & 0,021 & 0,267 & 0,048 \\
\hline$r_{\mathrm{d}}^{\prime \prime}$ en $\mathrm{mm}$ & 0,106 & 0,106 & 0,046 & 0,046 \\
\hline
\end{tabular}

de $1000 \mathrm{~kW}$ d'Odeillo. Leur principe, très simple, consiste à installer l'appareil photographique au niveau du concentrateur et à enregistrer l'image du soleil réfléchi dans un héliostat (Fig. 7a). Les clichés obtenus, dont l'un est reproduit figure $8 \mathrm{a}$, montrent des discontinuités des contours apparents du disque solaire d'une facette à l'autre. Or nous savons que ces discontinuités sont liées aux erreurs de réglage : à partir d'un point référencé $O_{r}$, nous déterminons sur une facette $F_{i}$ les erreurs en hauteur et azimut $h_{\mathrm{r} i}$ et $a_{\mathrm{r} i}$ en fonction de la position qu'occuperait, d'après son contour, le centre $\mathrm{C}_{i}$ du disque solaire pour la facette $\mathrm{F}_{i}$, par rapport à $\mathrm{O}_{\mathrm{r}}$. Le vecteur $\mathbf{O}_{\mathrm{r}} \mathbf{C}_{i}$ joue en effet le rôle du vecteur $\mathbf{T}$ de la relation (23) et il devient possible de remonter à $T_{r}$ pour 


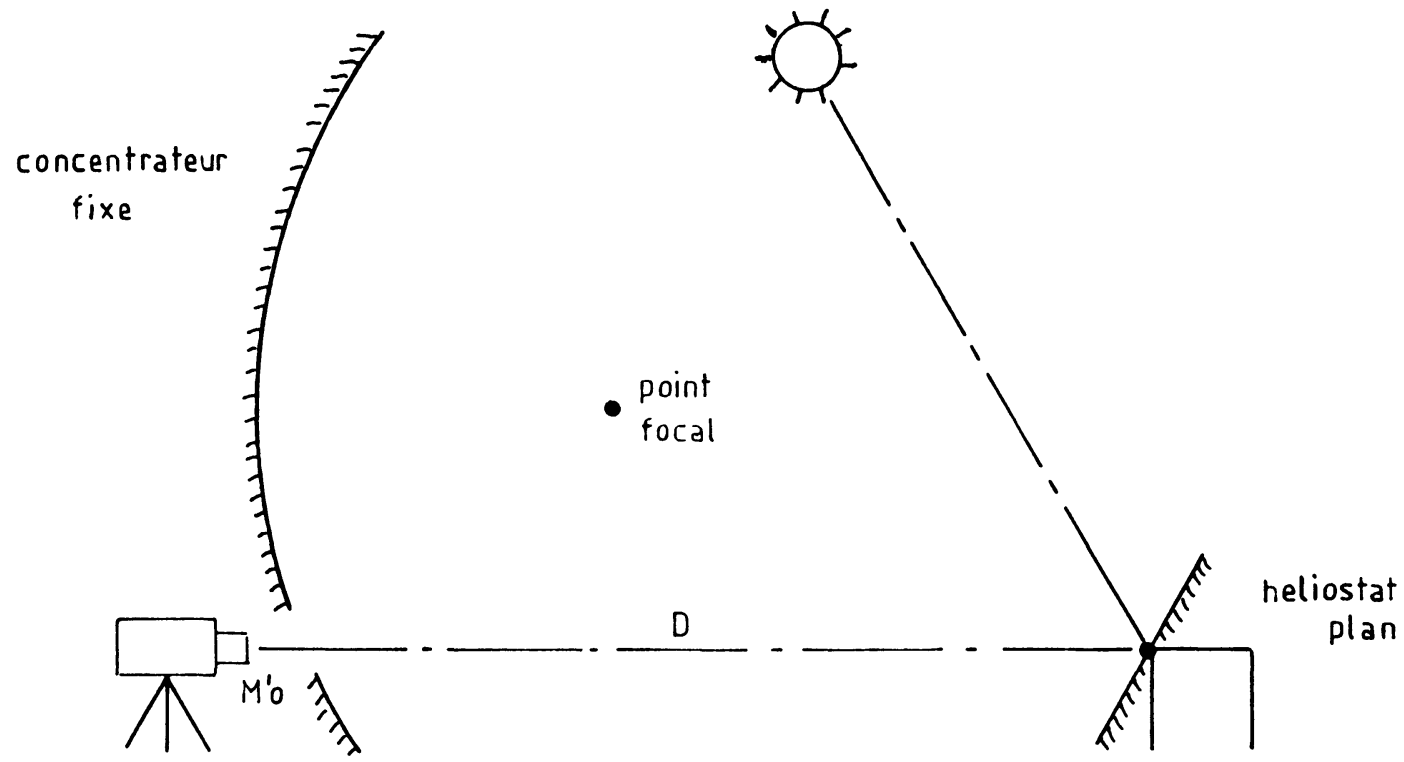

a)
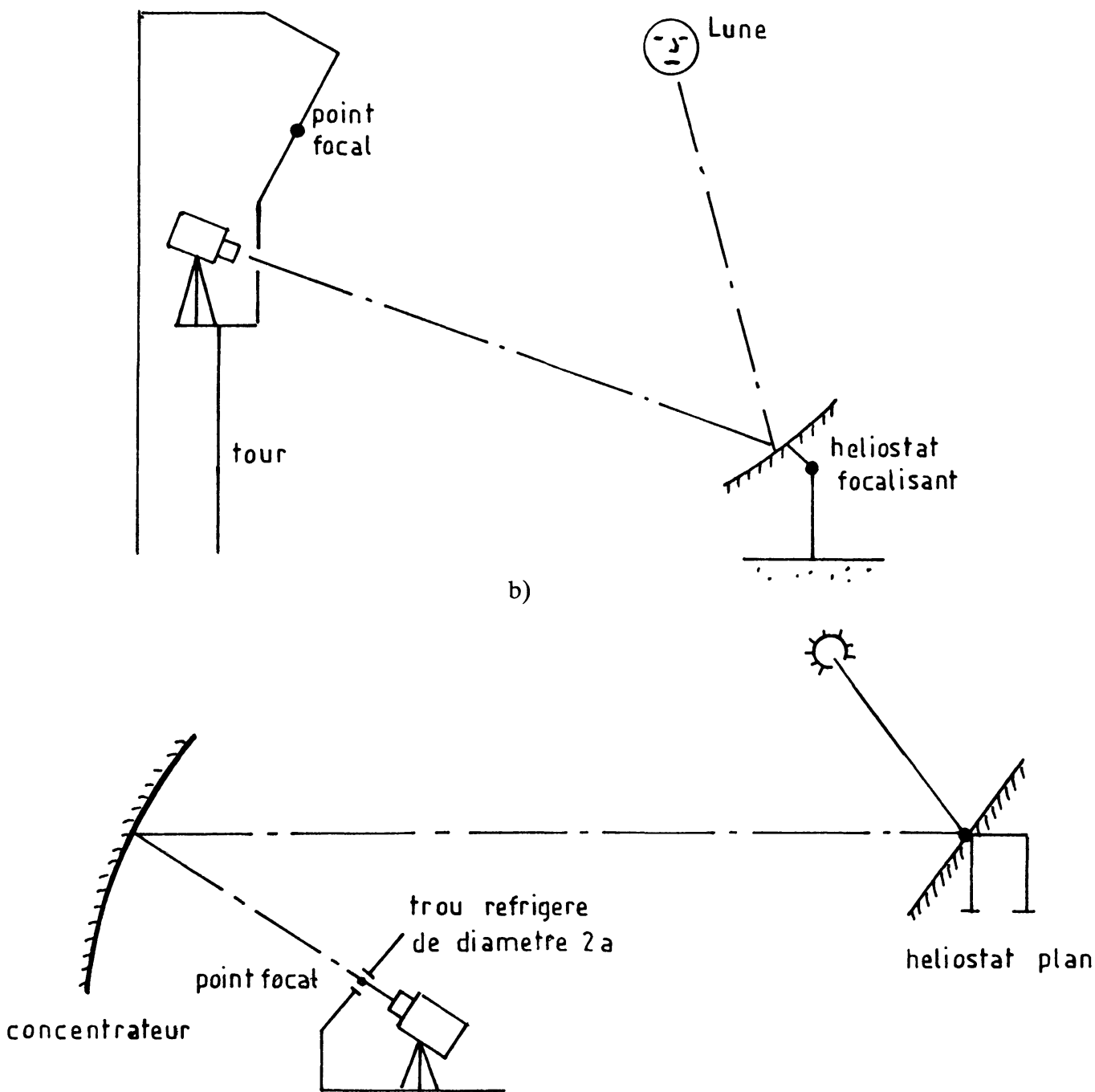

c)

Fig. 7. - Mesures effectuées sur un héliostat plan (a); sur un héliostat focalisant (b) ; sur un concentrateur ponctuel (c). [Sketches of principles for radiance measurements on a flat heliostat (a); on a focusing heliostat (b) ; on a double reflection point focusing concentrator (c).] 


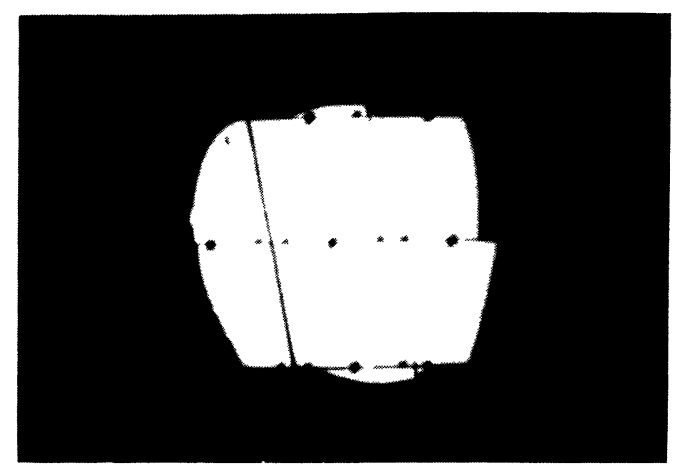

a)

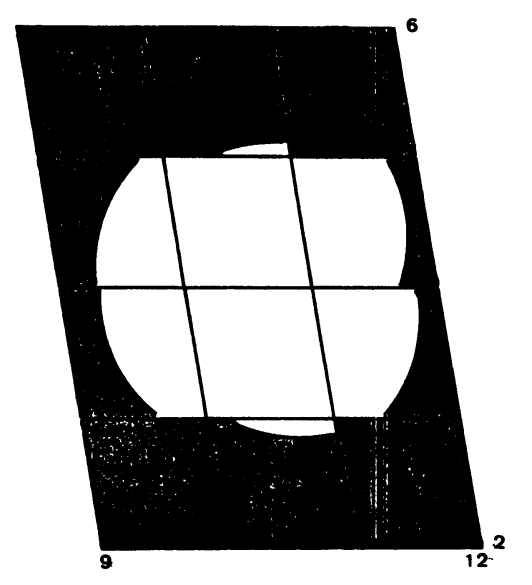

Hetostat 7

LE $10 / 9 / 85 \quad 1$ 10 418

COOROONEES W CONTRE

$r=0.799 \mathrm{M}$

$x=1.010 \mathrm{~N}$

EQRAIREKENT AU POINT $n^{\prime}$ $E=798.031$ VIR

FACTEUR DE REPEXION $R=0.7980$

b)

Fig. 8. - Répartitions de luminance observables sur un héliostat plan: (a) expérimentales ; (b) théoriques.

[Radiance patterns on a flat heliostat : (a) experimental ; (b) computer simulation.]

chaque facette $F_{i}$ (on rappelle que les vecteurs $\mathbf{T}_{\mathrm{p}}$ sont éliminés par moyennage sur les résultats). Une simulation numérique (Fig. 8b) nous permet ensuite de vérifier que ces erreurs, réintroduites dans notre code de calcul, reproduisent effectivement les répartitions de luminance photographiées.

Les résultats de cette première validation se sont révélés bons, ainsi qu'on peut en juger sur la figure 8. Dans le cas des héliostats plans du four de $1000 \mathrm{~kW}$ d'Odeillo, l'analyse statistique des erreurs de réglage en hauteur et en azimut a montré que celles-ci suivaient des lois normales indépendantes, d'écarts types respectifs égaux à 0,6 et $0,4 \mathrm{mrad}$, et de moyennes nulles. Ces résultats sont à mettre en rapport avec la méthode originale de réglage, qui est une méthode d'autocollimation au moyen d'un théodolite. On peut en conclure que la précision de cette méthode est très bonne, et vraisemblablement meilleure que celle qu'on peut attendre de la méthode classique des niveaux à bulle utilisée à Themis. Si l'on peut actuellement envisager de mettre en œuvre des procédures de réglage plus rapides, il ne sera pas aisé d'en trouver une qui soit plus performante.
Nous avons également procédé à une deuxième validation qui consiste à comparer le facteur de réflexion apparent à une distance $D_{i}$ de l'héliostat, mesuré au pyrhéliomètre, avec la valeur théorique obtenue par intégration numérique sur le cliché simulé. Une différence systématique de l'ordre de - $10 \%$ entre les valeurs expérimentale et théorique nous a amenés à introduire dans le modèle le relief réel des facettes, dont les mesures sont en cours, et qui semble légèrement différent de la forme plane idéale. Cela prouve que sur ces héliostats les défauts de surface sont loin d'être négligeables (vraisemblablement d'un ordre de grandeur comparable à celui des défauts de réglage), et qu'ils doivent être intégrés dans toute évaluation des performances énergétiques d'un héliostat plan.

Application AUX héliostats fOCAlisants. Ces mesures ont été réalisées sur le site de la centrale solaire expérimentale Themis, située à Targasonne. Le principe est sensiblement le même que pour les héliostats plans (Fig. 7b) : un appareil photographique est placé à une fenêtre de la tour, située à $12 \mathrm{~m}$ au-dessous de la chaudière, et vise différents héliostats focalisants programmés pour renvoyer les rayons lunaires sur la fenêtre. C'est donc la lune, dont le diamètre apparent diffère très légèrement de celui du soleil, qui est observée à travers les surfaces réfléchissantes. Un exemple de cliché est reproduit figure 9a. Les figures obtenues peuvent être approximées à des ellipses, dont le décalage par rapport à leur position idéale (tenant compte de la sphéricité de l'héliostat) est la somme de l'erreur de réglage et de l'erreur de pointage. Comme dans le cas de l'héliostat plan, cette dernière est éliminée sur chaque facette en soustrayant la moyenne des erreurs en hauteur et en azimut mesurées sur tout l'héliostat. Un programme de simulation permet ici aussi de vérifier que les erreurs déduites formeraient des répartitions de luminance conformes à celles du cliché étudié. Nous donnons figure $9 \mathrm{~b}$ la simulation correspondant au cliché reproduit. La deuxième validation consiste ici à mesurer des cartes de densité de flux avec la cible active, qui est un maillage géant de $32 \times 32$ photodiodes espacées de $22 \mathrm{~cm}$, et est destinée à l'appréciation individuelle des performances des héliostats focalisants. Nous donnons figure 10 trois exemples de cartes de flux, la première mesurée sur la cible active, la deuxième prédite en fonction des défauts de réglage obtenus par notre méthode et la troisième prédite en supposant ces erreurs nulles. On peut constater que la deuxième carte est plus proche de la carte mesurée que la troisième. Il apparaît dans ce cas précis que les performances de l'héliostat sont meilleures lorsqu'il est déréglé que s'il ne l'était pas. Ce résultat surprenant montre qu'ici les défauts de réglage ne sont pas aléatoires et peuvent s'expliquer 


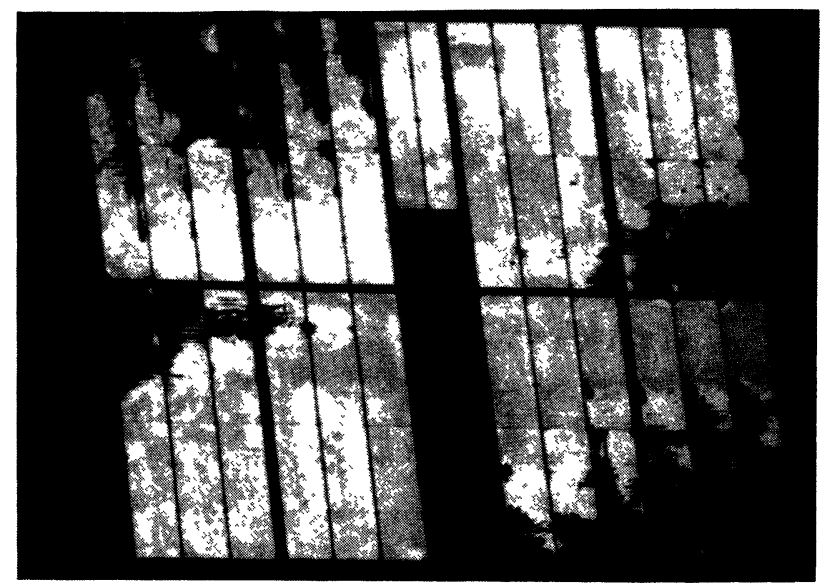

a)

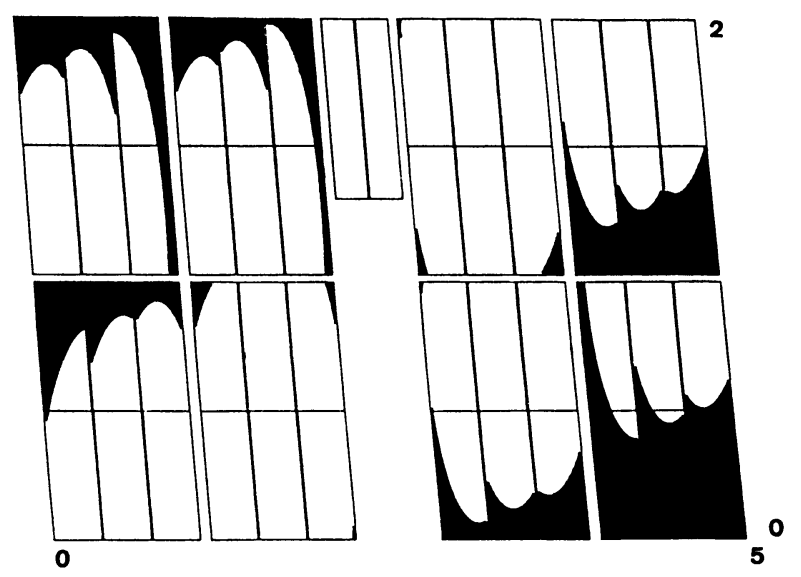

b)

Fig. 9. - Répartitions de luminance observables sur un héliostat focalisant : (a) expérimentales ; (b) théoriques.

[Radiance patterns on a focusing heliostat : (a) experimental ; (b) computer simulation.]

par le fait que cet héliostat ait été réglé sur cible pour une certaine position du soleil dans le ciel.

Par ailleurs, les défauts de surface des miroirs des héliostats focalisants Cethel III bis sont plus importants que ceux des héliostats plans du four de $1000 \mathrm{~kW}$ d'Odeillo, ce qui peut dans certains cas fausser l'estimation de leurs défauts de réglage. Il semble donc exclu, si on élimine les héliostats optimisés sur cible et ceux sur lesquels les défauts de surface sont prépondérants, de pouvoir se livrer à une analyse statistique des erreurs de réglage sur un grand nombre d'héliostats de la centrale Themis.

\section{CONTRÓLE DE SURFACES DE FACETTES DÉFORMÉES.}

- Cette étude a été menée sur le concentrateur paraboloïdal du four de $1000 \mathrm{~kW}$ d'Odeillo. Celui-ci est équipé de facette planes à l'origine, et déformées sous contrainte mécanique de manière à obtenir la focalisation optimale en tous points de la surface réfléchissante $[2,10]$. Il est intéressant de savoir si cette technique permet réellement de reproduire le relief de la surface localement paraboloïdale parfaitement stigmatique, ce relief permettant en théorie d'observer le centre du soleil sur toute la facette.

Un trou réfrigéré de diamètre $2 a=7 \mathrm{~mm}$ a donc été placé au foyer du concentrateur tandis qu'un héliostat était asservi sur le soleil (Fig. 7c). L'appareil photographique situé derrière le trou enregistre une séquence de clichés correspondant aux réglages suivants effectués sur la facette (Fig. 11) :

1) répartition de luminance observée initialement sur la facette (Fig. 11a);

2) répartition de luminance observée après élimination de la courbure initiale de la facette. Celle-ci est alors considérée comme ayant retrouvé sa forme plane d'origine, et le décalage entre le centre de l'image observée et le centre de la facette est lié à son erreur de réglage à l'origine (Fig. 11b) ;

3) répartition de luminance observée après correction de l'erreur originale de réglage (Fig. 11c);

4) répartition de luminance optimale observée après réglage de la courbure de la facette (Fig. 11d). Le gain en concentration global peut être estimé en faisant le rapport des surfaces brillantes après et avant cette séquence d'opérations.

$\mathrm{Au}$ vu du résultat final, on peut constater que les facettes déformées sont très loin d'approcher la surface localement paraboloïdale, ou même la surface sphérique. Toutefois, bien que cette procédure inédite d'optimisation semble plus rapide et performante que la méthode de réglage utilisée lors de la mise en service du concentrateur, elle ne donne qu'un diagnostic pour l'évaluation des performances des miroirs, puisqu'il n'est pas possible, à l'aide d'un appareil photographique, d'obtenir les courbes isoluminance formées sur la facette. De plus, la source lumineuse est l'image solaire réfléchie par un héliostat, et présente donc elle-même de sérieuses discontinuités. Le développement d'une méthode permettant de quantifier les défauts de surface des facettes réflectrices est en cours. Son principe consiste à mesurer les déformations apparentes, à travers la facette placée en autocollimation, de l'image d'une grille régulière située dans le plan du trou de diamètre $2 a$.

En fait il s'agit encore ici d'une méthode analogue à celles qui ont été décrites plus haut, la source lumineuse naturelle (soleil ou lune) utilisée jusqu'alors étant remplacée par une source artificielle.

\section{Conclusion.}

Les erreurs de réglage des facettes réflectrices sont des paramètres indispensables à l'évaluation des performances énergétiques des surfaces qu'elles forment. Les méthodes que nous avons mises au point, bien que très simples et de résolution moyenne, 


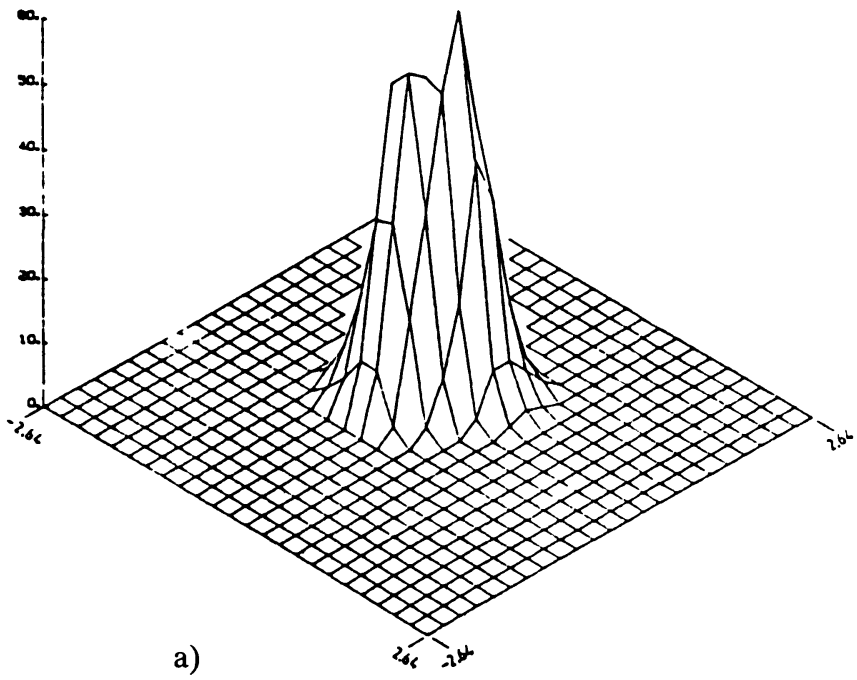

mestat 3

LE $201212185 \quad 113,48,0$

comx $=60.3350$

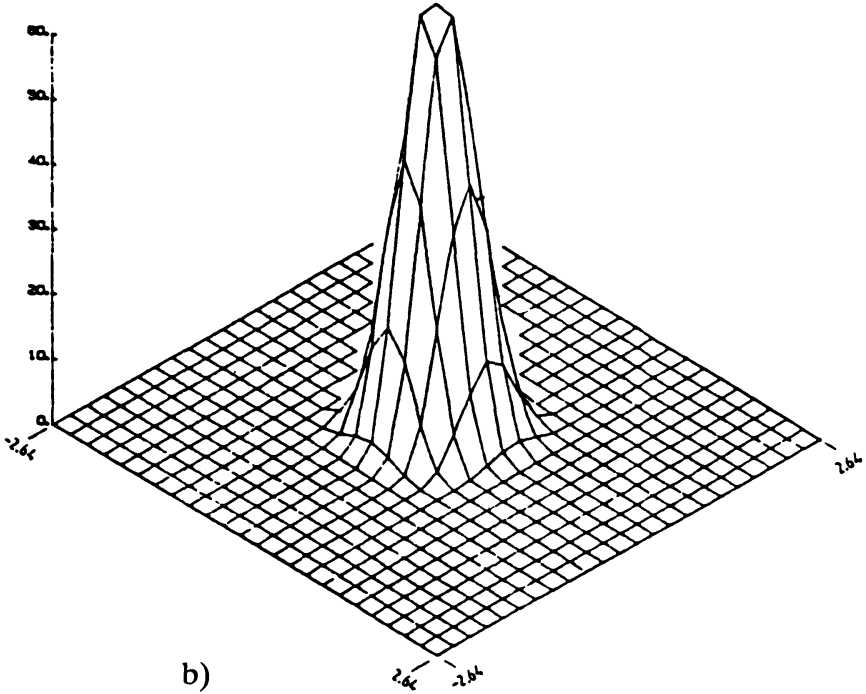

helostat 3

LE $20112 / 85113,480$

CuLCx $=84.1078$

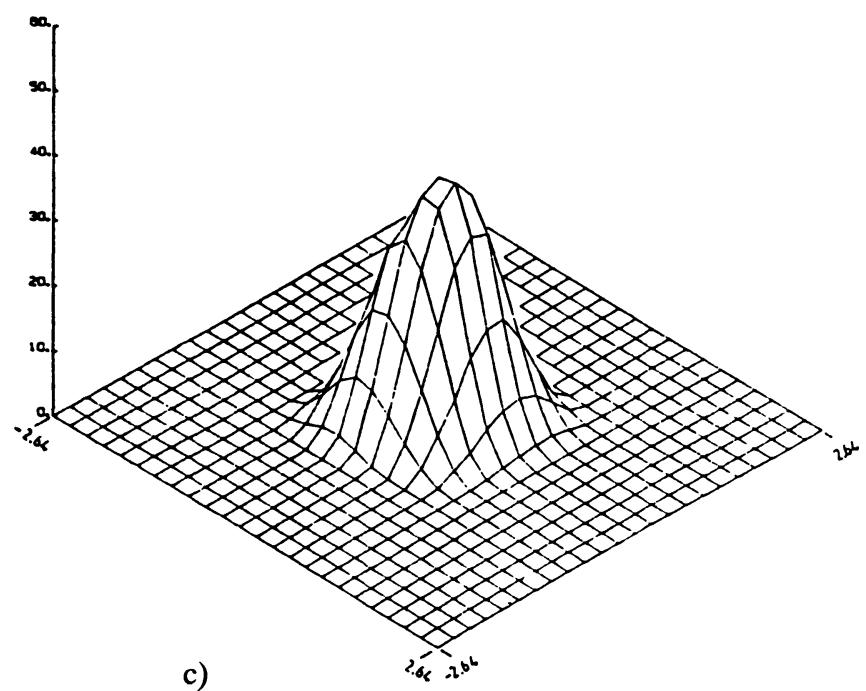

helosiat 3

LE $20 / 12 / 85 \quad 1: 3,48,0$

coux= 36.7314

Fig. 10. - Répartitions de densité de flux formées par un héliostat focalisant : (a) expérimentales ; (b) théoriques avec erreurs de réglage; (c) théoriques sans défauts de réglage.

[3D maps of irradiance from a focusing heliostat : (a) experiment ; (b) computer simulation with the alignment errors ; (c) computer simulation without the alignment errors.] 


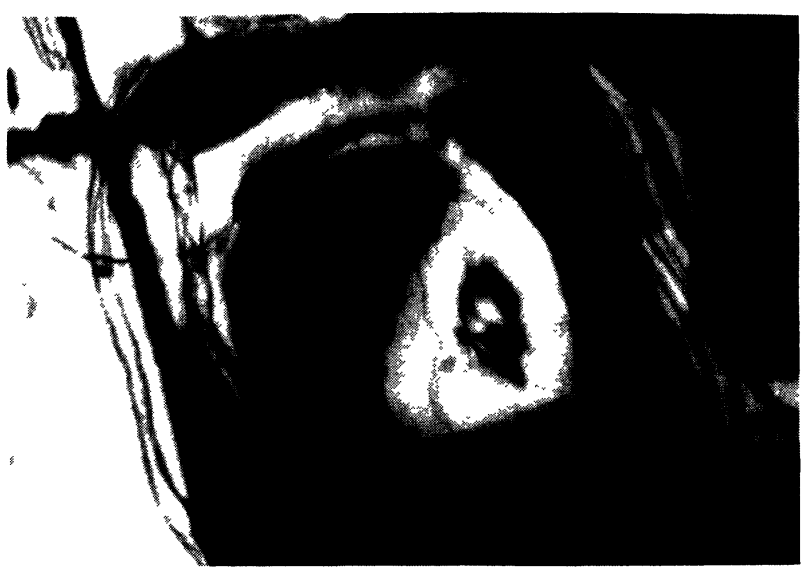

a)

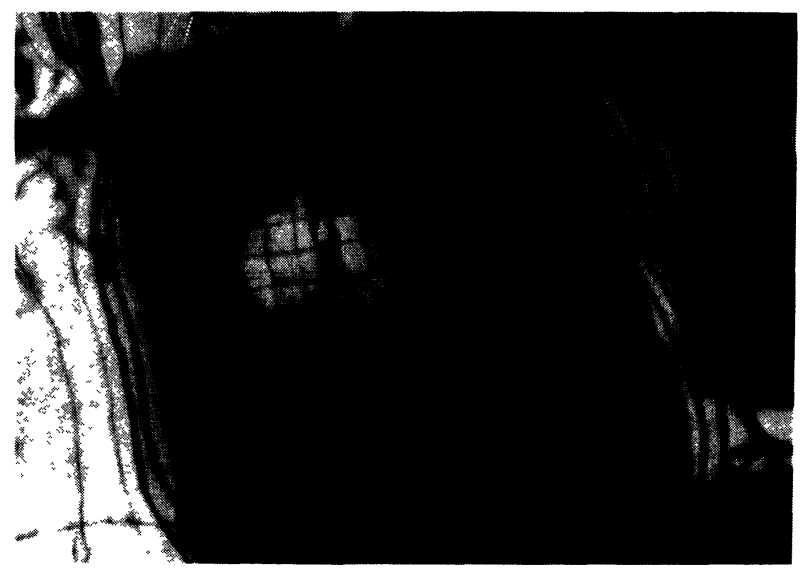

b)

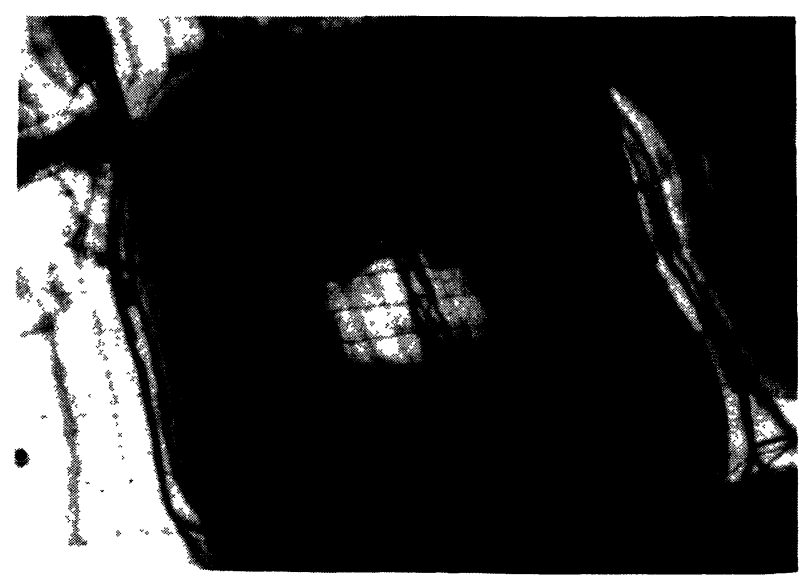

c)

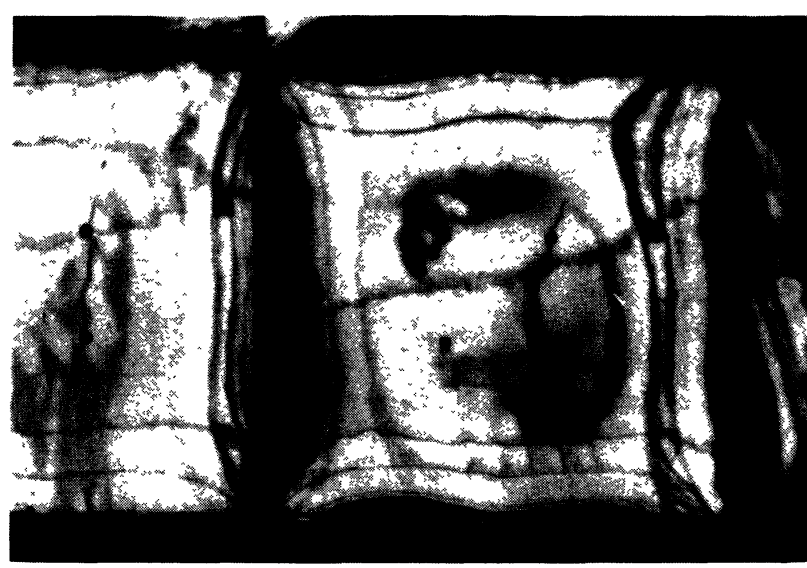

d)

Fig. 11. - Répartitions de luminance vue du foyer du concentrateur de $1000 \mathrm{~kW}$.

[Radiance patterns from the focus of the $1 \mathrm{MW}$ concentrator.]

constituent une base solide pour la réalisation d'un outil de diagnostic des grandes installations solaires. Il apparaît en effet que l'appareil photographique ne permet d'appréhender que les contours extérieurs des répartitions de luminance, c'est-à-dire les limites entre les zones éclairées et les zones sombres, qui correspondent aux contours du disque solaire. En le remplaçant par exemple par une caméra numérique [1], et en jouant sur l'assombrissement aux bords du disque solaire, il deviendrait possible d'obtenir des courbes iso-luminance à l'intérieur de zones complètement éclairées par le soleil. La réalisation d'un tel outil serait certes une étape importante pour la connaissance des installations solaires. Adapté convenablement, il pourrait permettre la mesure instantanée de défauts de réglage ou de pointage d'héliostats, de défauts de surface de facettes, et de leurs variations en fonction de facteurs tels que la vitesse du vent, la gravité, la température... Sans parler de transmission d'images en temps réel, qui permettraient l'optimisation in situ des surfaces réflectrices. Une nouvelle génération de méthodes de réglage des installations, plus souples, plus performantes, pourrait alors voir le jour.

\section{Bibliographie}

[1] Brumleve T. D., Gibson J. C., Measurement Challenges in Solar Central Receiver Systems, 7th Energy Technology Conf.

[2] Le Phat Vinh A., Répartition de l'énergie solaire au foyer d'un miroir parabolique. Calculs et étude expérimentale, thèse de Doctorat d'Université Journal des Recherches du CNRS 57 (1961).
[3] Alcayaga O., Contribution à l'étude de la répartition de la densité de flux énergétique dans l'espace focal d'un système concentrateur de rayonnement solaire, thèse de Doctorat de $3^{\mathrm{e}}$ cycle (Poitiers) 1977.

[4] Biggs F., Vittitoe C. N., The Helios model for the optical behavior of reflecting solar concentrators, SAND 76-0347 1979. 
[5] Pettit R. B., Characterization of the reflected beam profile of solar mirror materials, Sol. Energy 19 (1977) 733-741.

[6] Avellaner J. A., Optical characterization of the facets of a heliostat, Revue Phys. Appl. 15 (1980) 169-173.

[7] MACFEe R. H., Power collection reduction by mirror surface non-flatness and tracking error for a central receiver solar power system, Appl. Opt. 14 (1975) 1493-1502.

[8] Hugues R. O., Effects of tracking errors on the performance of point focusing solar collectors, Sol. Energy 24 (1980) 83-92.
[9] Henault F., Concentration du rayonnement solaire par simple et double réflexions: contribution aux méthodes de réglage et de contrôle des surfaces réflectrices à facettes, thèse de Docteur en sciences, Université Paris XI (Orsay) 1987.

[10] Trombe F., Le Phat Vinh A., Etudes théoriques sur la concentration du rayonnement solaire et procédés de mesure. Réalisations pratiques de la concentration du rayonnement solaire, Cahiers AFEDES 3 (novembre 1971). 